\title{
RENDA BRUTA DE MÚLTIPLOS PRODUTOS DE MADEIRA EM POVOAMENTOS DE Pinus taeda SUBMETIDOS A DIFERENTES DENSIDADES DE PLANTIO E REGIMES DE DESBASTE: ABORDAGEM EXPERIMENTAL
}

\author{
Gross Profit of Pinus taeda Stands Growing Under Different \\ Densities and Thinning Regimes: an Experimental Approach \\ on Multi-products
}

\author{
Carlos R. Sanquetta ${ }^{1}$ \\ Alba V. Rezende ${ }^{2}$ \\ Débora Gaiad ${ }^{3}$ \\ Luciano B. Schaaf ${ }^{3}$ \\ Ana C. Zampier ${ }^{3}$ \\ Julio E. Arce ${ }^{4}$ \\ Ana Paula Dalla Côrte ${ }^{4}$
}

\section{Resumo}

Este artigo avalia experimentalmente o efeito de diferentes densidades de plantio e regimes de desbaste no Valor Presente Bruto da receita (VPB), advinda da produção de múltiplos produtos de madeira em povoamentos de Pinus taeda. Os dados advêm de um experimento implantado no município de Jaguariaíva, Paraná, onde foram avaliados 5 espaçamentos: 2,5 x 1,2 m; 2,5 x 2,0 m; 2,5 x 2,8 m; 2,5 x 3,6 m e 2,5 x 4,4 $\mathrm{m}$. Cinco regimes de desbaste foram confrontados: corte final aos 15 anos, sem desbaste; corte final aos 20 anos, sem desbaste; desbaste sistemático na $6^{\text {a }}$ linha aos 9 anos e corte final aos 20 anos; desbastes seletivos por baixo, com redução de 50\% do número de árvores aos 9 e 15 anos e corte final aos 20 anos; e desbaste

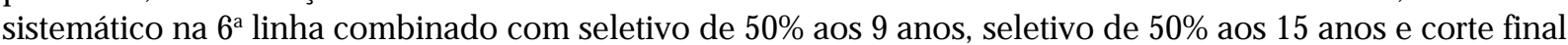
aos 20 anos. $\mathrm{O}$ experimento fatorial foi analisado por meio de ANOVA e pelo teste de Tukey. Os resultados mostram diferenças significativas a $1 \%$ entre os espaçamentos e entre os regimes de desbaste, sendo a interação entre ambos também significativa. Evidenciou-se que, para a variável VPB, a combinação ideal é o emprego de um espaçamento menor, isto é, povoamento mais denso, como 2,5 x 1,2 m, combinado com a realização de desbastes mais freqüentes, aos 9 e aos 15 anos, preferivelmente com a adoção de desbaste sistemático na primeira intervenção. Essa combinação foi a que determinou a maior renda bruta no presente estudo, a qual diferiu estatisticamente dos demais tratamentos. Isto revela a importância de proceder análise experimental dos regimes de manejo, permitindo, assim, decidir com melhor juízo a opção que gera a maior renda bruta.

Palavras-chave: Desbaste; Espaçamento; Simulação; Sítio; Valor presente.

1 Professor do Departamento de Ciências Florestais da UFPR, Bolsista do CNPq.

2 Professora do Departamento de Engenharia Florestal da UnB, Doutoranda do Curso de Pós-Graduação em Engenharia Florestal da UFPR.

3 Mestrando do Curso de Pós-Graduação em Engenharia Florestal da UFPR.

4 Professor do Departamento de Ciências Florestais da UFPR. 


\section{Abstract}

This paper evaluates the statistical effects of five different thinning regimes in combination with five initial plant spacing in loblolly pine plantations on the gross profit of roundwood multiproduct yield (VPB). The data came from an experimental plantation located in Jaguariaiva, Parana State, southern Brazil. The plant spacing evaluated were: $2.5 \times 1.2 \mathrm{~m} ; 2.5 \times 2.0 \mathrm{~m} ; 2.5$ × $2.8 \mathrm{~m} ; 2.5$ x $3.6 \mathrm{~m}$ e $2.5 \times 4.4 \mathrm{~m}$. The thinning regimes compared were: a single final cut at age 15 without thinning; a single final cut age 20 without thinning; systematic thinning along the $6^{\text {th }}$ planting line at age 9 and final cut at age 20; selective thinning for below of $50 \%$ of the trees in the stand at ages 9 and 15 and final cutat age 20 ; and systematic cut along the $6^{\text {th }}$ planting line combined with selective thinning at age 9 for below of $50 \%$ of the trees in the stand, selective thinning for below of $50 \%$ of the trees in the stand at age 15 and final cut at age 20. The factorial experiment was analyzed by means of ANOVA and the test of Tukey. The results revealed significant differences $(p<0.01)$ among initial plant spacing and thinning regimes. The interaction between them was also statistically significant. The results demonstrated that, in terms of VPB, the best combination of spacing and thinning regime would be obtained with less dense stands (close initial spacing), as $2.5 \times 1.2 \mathrm{~m}$, together with frequent thinning; at age 9 and 15, preferably with a systematic intervention at age 9 . This combination led to the treatment of the highest gross profit in the present study, which was statistically different from all other tested treatments. Further studies are necessary to clarify the effect of these costs on management regime selection.

Keywords: Thinning; Spacing; Simulation; Site; Present value.

\section{Introdução}

Planejar o uso dos recursos florestais visando tanto a atender as necessidades do mercado consumidor, como também obter a maximização dos lucros, é um desafio de qualquer empreendimento, o que também é o fito das empresas florestais. Atualmente, várias empresas têm dado ênfase ao uso múltiplo da madeira, o que segundo Acerbi Jr. et al. (1999) proporciona uma maior remuneração da floresta como um todo, havendo melhor aproveitamento de cada árvore e uma menor dependência das oscilações do mercado em segmentos isolados.

No Sul do Brasil, o setor florestal tem como principal cultura plantada o gênero Pinus, que fornece matéria-prima para vários mercados, como o de papel, serrados, chapas, entre outros. Particularmente para este gênero, o crescimento e a produtividade das plantações sofrem grande influência da densidade inicial de plantio e dos desbastes realizados, afetando diretamente a obtenção dos múltiplos produtos.

Os povoamentos de Pinus possuem a característica de poder fornecer madeira para diversas classes de utilização. De acordo com a bitola e a qualidade das toras produzidas, tanto o mercado-alvo quanto o valor da matéria-prima é distinto. A produção contínua de madeira para diversos usos ao longo da vida de um povoamento florestal depende da série de operações realizadas na floresta, como, por exemplo, a escolha da espécie, procedência, espaçamento inicial de plantio, tratamentos silviculturais, desbastes e poda (SCOLFORO; MAESTRI, 1998).

Segundo Smith e Strub (1991), espaçamentos mais amplos devem ser utilizados quando o objetivo é a produção de madeira para serraria em rotações curtas, sem a realização de desbastes, pois resultam em árvores de maiores dimensões com redução dos custos de exploração. Já para o caso de produção conjunta de madeira, processo (pasta celulósica) e serraria, espaçamentos menores podem ser vantajosos, desde que haja mercado para a madeira retirada nos desbastes, pois a realização destes envolve custos elevados.

A baixa remuneração da matéria-prima proveniente de desbastes realizados em povoamentos excessivamente densos tem motivado uma forte tendência de uso de espaçamentos mais amplos. Operações de desbaste são dispendiosas, e, portanto, a relação custo/benefício desta operação deve ser cuidadosamente avaliada. Os desbastes que aparentemente envolvam uma perda financeira só deverão ser realizados se houver uma compensação no retorno final da rotação (SHEPHERD, 1986).

O regime de desbaste e a idade de corte final devem ser definidos de acordo com 0 objetivo da produção madeireira e a escolha da densidade de plantio deve estar intimamente relacionada com o regime de desbaste a ser utilizado (GOMES et al., 1998). As ferramentas existentes para a simulação de crescimento e 
produção podem ser utilizadas no planejamento florestal para verificação da produção esperada em idades futuras, em diferentes condições de sítio e densidade (GOMES, 1999).

Vários autores se dedicaram a desenvolver e utilizar sistema de simulação ou outras técnicas de programação matemática para eleição de regimes de manejo em povoamentos de Pinus, entre os quais Ahrens (1992), Volpi (1997), Oliveira et al. (1998), Gomes (1999) e Acerbi et al. (1999). Em todos os trabalhos previamente mencionados, a indicação do melhor regime foi feita com base num único valor, em geral representando o melhor resultado econômico obtido. Igualdades ou diferenças estatísticas decorrentes das oscilações internas dos tratamentos não foram levadas em consideração.

O objetivo deste trabalho é avaliar estatisticamente a renda bruta decorrente da produção volumétrica dos múltiplos usos da madeira (celulose, serraria e laminação), nos diferentes regimes de desbaste e espaçamentos de plantio em plantios experimentais de Pinus taeda. Especial referência é dada não apenas à melhor média de um determinado tratamento, mas também à significância experimental entre as médias dos diversos tratamentos de manejo analisados.

\section{Material e métodos}

Os dados utilizados para este estudo são provenientes de um experimento de Pinus taeda, localizado no Projeto 26, talhão 11, Fazenda Lageado, município de Jaguariaíva-PR, de propriedade da empresa PISA Florestal.

O experimento foi implantado no período de 28 de outubro a 09 de novembro de 1987, segundo um delineamento em blocos casualizados com seis repetições, instaladas de forma contínua, em um total de $29.692 \mathrm{~m}^{2}$, incluindo a bordadura. A área ocupada pelos seis blocos é de $25.920 \mathrm{~m}^{2}$, ou seja, $4.320 \mathrm{~m}^{2} /$ bloco. Cada bloco corresponde a um sítio diferente, sendo o bloco 1 o de menor valor médio de índice de sítio e 0 bloco 6 o de maior valor.

As coletas de dados foram feitas em seis idades: 3,5 anos (maio/91), 4,5 anos (maio/92), 5,92 anos (outubro/93), 6,92 anos (outubro/94), 7,67 anos (julho/95) e 8,75 anos (agosto/96). Nas primeiras coletas, foram medidas apenas as alturas totais das árvores da parcela. Quando as árvores atingiram 1,30 $\mathrm{m}$, passou-se a medir também, a CAP (circunferência à altura do peito).

Em cada bloco foram testados cinco espaçamentos (TABELA 1). Em cada parcela de cada bloco, foram mensuradas apenas as árvores presentes na sua área útil.

\section{TABEIA 1 - Tratamentos analisados em um experimento de manejo de Pinus taeda.}

Table 1 - Treatments analyzed in a management experiment of loblolly pine.

\begin{tabular}{ccccc}
\hline & \multicolumn{3}{c}{ Area daParcela $\left(\mathrm{m}^{2}\right)$} \\
Espaçamento $(\mathrm{m})$ & Árvores/ha & \multicolumn{2}{c}{ Plot area $\left(\mathrm{m}^{2}\right)$} & Plantas Úteis \\
Spacing $(m)$ & Trees $/$ ha & Total & Útil & Number of plants \\
& & Total & Useful \\
\hline $1-2,5 \times 1,2$ & 3333 & 630 & 234 & 78 \\
$2-2,5 \times 2,0$ & 2000 & 630 & 210 & 42 \\
$3-2,5 \times 2,8$ & 1428 & 810 & 315 & 45 \\
$4-2,5 \times 3,6$ & 1111 & 990 & 378 & 42 \\
$5-2,5 \times 4,4$ & 909 & 1260 & 440 & 40 \\
\hline
\end{tabular}


A partir dos dados coletados foram analisados também cinco regimes de desbaste, mediante simulações, com o objetivo de definir o manejo ideal dentro de cada espaçamento. Os regimes de desbaste variaram em função do método de desbaste (seletivo, sistemático ou livre), da redução na densidade e idade de corte raso. Foram avaliados os seguintes regimes de desbaste:

1. Corte final aos 15 anos, sem desbaste (CF $15 \mathrm{~s} /$ desb);

2. Corte final aos 20 anos, sem desbaste (CF $20 \mathrm{~s} / \mathrm{desb})$;

3. Desbaste sistemático na $6 .{ }^{\mathrm{a}}$ linha aos 9 anos e corte final aos 20 anos (Sist 9 + CF 20);

4. Desbastes seletivos por baixo, com redução de 50\% do número de árvores aos 9 e 15 anos e corte final aos 20 anos (Sel 9, Sel $15+\mathrm{CF}$ 20);
5. Desbaste sistemático na $6^{\mathrm{a}}$ linha combinado com seletivo de $50 \%$ aos 9 anos, seletivo de $50 \%$ aos 15 anos e corte final aos 20 anos (Sist+Sel 9, Sel $15+$ CF 20).

As simulações foram realizadas mediante a utilização do programa SISPINUS, que permite, além da simulação de desbastes e do crescimento e produção anual do povoamento, o sortimento de madeira por classe diamétrica (OLIVEIRA, 1995). O sistema utiliza como variáveis de entrada o número de árvores por hectare (N/ha) e a área basal em $\mathrm{m}^{2} /$ ha $(\mathrm{G} / \mathrm{ha})$ de cada tratamento e o índice de sítio (IS) de cada bloco. Neste estudo, a prognose de produção foi realizada a partir de dados obtidos na idade de 9 anos, em condições distintas de sítio. Os dados utilizados para a efetivação da simulação são apresentados na Tabela 2.

\section{TABELA 2 - Dados básicos de um plantio experimental de Pinus taeda usados para fins de simulação no programa SISPINUS.}

Table 2 - Basic data of loblolly pine experimental stand utilized to start the simulation on the SISPINUSsoftware.

\begin{tabular}{|c|c|c|c|c|c|c|c|c|c|c|c|c|}
\hline \multirow{2}{*}{$\begin{array}{c}\text { Espaçamento } \\
\text { Spacing } \\
\text { (m) }\end{array}$} & \multicolumn{2}{|c|}{$\begin{array}{l}\text { Bloco/Block } 1 \\
\text { IS/Site }=16,6\end{array}$} & \multicolumn{2}{|c|}{$\begin{array}{l}\text { Bloco/Block } 2 \\
\text { IS } / \text { Site }=17,4\end{array}$} & \multicolumn{2}{|c|}{$\begin{array}{l}\text { Bloco/Block } 3 \\
I S / \text { Site }=18,4\end{array}$} & \multicolumn{2}{|c|}{$\begin{array}{l}\text { Bloco/Block } 4 \\
\text { IS/Site }=18,1\end{array}$} & \multicolumn{2}{|c|}{$\begin{array}{l}\text { Bloco/Block } 5 \\
\text { IS/Site }=18,7\end{array}$} & \multicolumn{2}{|c|}{$\begin{array}{l}\text { Bloco/Block } 6 \\
I S / \text { Site }=18,7\end{array}$} \\
\hline & G/ha & N/ha & G/ha & N/ha & G/ha & N/ha & G/ha & N/ha & G/ha & N/ha & G/ha & N/ha \\
\hline $1-2,5 \times 1,2$ & 44,8 & 3333 & 47,3 & 3419 & 48,5 & 3205 & 47,0 & 3248 & 52,6 & 3248 & 50,0 & 3291 \\
\hline $2-2,5 \times 2,0$ & 38,2 & 2000 & 41,1 & 2095 & 42,1 & 1952 & 40,4 & 2000 & 48,7 & 2048 & 49,2 & 2190 \\
\hline $3-2,5 \times 2,8$ & 34,0 & 1492 & 35,9 & 1429 & 38,6 & 1429 & 39,1 & 1429 & 39,1 & 1429 & 36,9 & 1333 \\
\hline $4-2,5 \times 3,6$ & 31,0 & 1111 & 28,7 & 1164 & 33,2 & 1111 & 35,3 & 1138 & 35,9 & 1138 & 38,0 & 1217 \\
\hline $5-2,5 \times 4,4$ & 28,8 & 909 & 29,0 & 932 & 30,4 & 932 & 31,5 & 909 & 31,5 & 955 & 31,2 & 955 \\
\hline
\end{tabular}

IS : Índice de Sítio médio, correspondente à altura média das árvores dominantes (m) aos 9 anos de idade / Site index at age 9 yr.

G/ha: Área basal (m²/ha) aos 9 anos de idade / Basal area at age 9 yrs

N/ha: Número de árvores/ha aos 9 anos de idade / Number of trees per hectare at age 9 yrs.

Pelo programa SISPINUS foram gerados os sortimentos em volume de madeira com casca, para celulose, serraria e laminação, utilizando toras de $2,40 \mathrm{~m}$ de comprimento e diâmetros variando de 8 a $18 \mathrm{~cm}$ para celulose, 18 a $25 \mathrm{~cm}$ para serraria e diâmetros maiores que $25 \mathrm{~cm}$ para laminação. As equações de volume e de índice sítio utilizadas neste estudo foram as já ajustadas para Pinus taeda do próprio SISPINUS (OLIVEIRA, 1995).

Os resultados da distribuição diamétrica prognosticados (valor central de cada classe de DAP, altura média correspondente e número de árvores por hectare estimado para cada classe) foram utilizados para estimar os volumes totais com casca de 
cada sortimento por hectare na idade desejada. As mesmas equações utilizadas para estimar os volumes aos 9 anos de idade foram usadas para as estimativas nas idades futuras (para o valor central de cada classe de DAP, fomecido pelo SISPINUS). Multiplicando-se este volume pelo número de árvores de cada classe (prognosticado pelo SISPINUS), obteve-se o volume total com casca de cada sortimento por hectare por classe de DAP. A soma dos volumes por hectare em todas as classes forneceu as variáveis necessárias para este estudo, ou seja, os volumes com casca para celulose, laminação e serrania.
Este estudo não tem como objetivo realizar uma avaliação econômica profunda dos tratamentos (regimes de manejo e espaçamentos), mas apresentar uma abordagem experimental sobre a variável renda bruta advinda deles. Para realizar esta avaliação, inicialmente foi calculado o valor, em reais, de cada volume de madeira com casca, obtido para cada sortimento em cada regime de manejo. Na Tabela 3, encontram-se os valores de mercado em reais dos preços de venda dos diferentes produtos considerados.

\section{TABELA 3 - Valores em reais dos preços de cada produto no mercado.}

Table 3 - Stumpage prices of each product in the region.

\begin{tabular}{ccc}
\hline Produtos Products & $\begin{array}{c}\text { Bitola }(\mathrm{cm}) \\
\text { Diameter }(\mathrm{cm})\end{array}$ & $\begin{array}{c}\text { Preços de Venda }\left(\mathrm{R} \$ / \mathrm{m}^{3}\right)^{*} \\
\text { Stumpage prices }\left(R \$ / \mathrm{m}^{3}\right)\end{array}$ \\
\hline Celulose Pulp & 8 a 18 & 13,68 \\
Serraria Sawntimber & 18 a 25 & 22,16 \\
Laminação Veneer & $>25$ & 33,45 \\
\hline
\end{tabular}

* Fonte / Source: PISA Florestal S.A.

Como critério de avaliação, adotou-se o Valor Presente Bruto, dado por:

$$
V P B=\sum_{t=0}^{n} \frac{C_{t}}{(1+i)^{t}}
$$

onde:

VPB = Valor Presente Bruto do fluxo de caixa futuro $(\mathrm{R} \$ / \mathrm{ha})$;

$\mathrm{C}_{\mathrm{t}}=$ Fluxo de caixa líquido no período $\mathrm{t}$;

$\mathrm{n}=$ número de anos envolvidos;

$\mathrm{i}=$ taxa anual de desconto.

Neste estudo, considerou-se uma taxa de juros de $6 \%$ a .a.

O valor referencial da moeda em 22/

05/2000 é de $\mathrm{R} \$ 1,85=$ US\$ 1.00 .
Após a obtenção do valor presente bruto de cada tratamento, procedeu-se uma análise de variância preliminar com os 6 blocos e os 25 tratamentos (espaçamentos, regimes de manejo e suas interações), considerando um nível de significância de $1 \%$, com o objetivo de verificar se os tratamentos apresentavam efeitos diferentes sobre estes valores.

Baseado na significância dos tratamentos, realizou-se o desdobramento deles com o objetivo de avaliar o efeito dos regimes de manejo, dos espaçamentos e da interação destes dois fatores, no valor presente bruto (VPB).

O teste de Tukey foi aplicado para comparar as médias dos efeitos que apresentaram diferença significativa a $5 \%$ pelo teste F. 


\section{Resultados e discussão}

Antes de realizar a análise estatística, 0 teste de Bartlett foi utilizado para testar a homogeneidade de variâncias dos dados a um nível de significância de 5\% (DRAPPER; SMITH, 1980;
SOKAL; ROLF, 1981). A partir do teste de Bartlett, verificou-se que os dados apresentavam variâncias homogêneas, não sendo necessário realizar a transformação dos dados. A Tabela 4 mostra os resultados da análise de variância para os valores de VPB da produção total de madeira.

TABELA 4 - Análise de variância do VPB em povoamentos experimentais de Pinus taeda. Table 4 - Analysis of variance of gross profit (VPB) in a loblolly pine experimental stand.

\begin{tabular}{lc|c}
\hline \multicolumn{1}{c|}{$\begin{array}{l}\text { Fonte de Variação } \\
\text { Source of variation }\end{array}$} & $\begin{array}{c}\text { Graus de } \\
\text { Liberdade }\end{array}$ & $\begin{array}{c}\text { Quadrados Médios do VPB (R } \$ / \text { ha }) \\
\text { Mean squared }(R \$ / h a)\end{array}$ \\
\hline Bloco & $d f$ & $4.683 .533,24 * *$ \\
Tratamentos & 5 & $487.695,80^{* *}$ \\
- Espaçamento & 24 & $711.990,14^{* *}$ \\
- Desbaste & 4 & $1.135 .210,29 * *$ \\
- Espaçamento x Desbaste & 4 & $269.743,59^{* *}$ \\
Resíduo & 16 & $44.155,32$ \\
\hline
\end{tabular}

**Significativo ao nível de $1 \%$ de probabilidade $(p<0.01)$

$\mathrm{CV} \%=6,67$ (coefficient of variation)

Média $=3.150,25\left[\mathrm{~m}^{3} / \mathrm{ha}\right]$ (mean)

Observa-se na Tabela 4 que existem diferenças significativas ao nível de $1 \%$ de probabilidade pelo teste $\mathrm{F}$, para os fatores espaçamento e regime de desbaste, indicando que estes apresentam efeitos diferentes sobre os valores em reais do VPB referente à produção de madeira dos múltiplos produtos considerados. Observa-se, ainda, que o efeito da interação espaçamento x desbaste tam- bém foi significativo, indicando, a princípio, que os espaçamentos diferem internamente entre os regimes de desbaste e vice-versa.

As médias dos valores em reais do VPB da produção total de madeira, que gravitam em torno de $\mathrm{R} \$ 3.000,00$ a $\mathrm{R} \$ 3.300,00 / \mathrm{ha}$, foram comparadas pelo teste de Tukey a $5 \%$ de probabilidade e os resultados estão apresentados na Tabela 5. 
TABELA 5 - Médias do VPB de múltiplos produtos de madeira de povoamentos experimentais de Pinus taeda, em cinco diferentes tipos de espaçamento e cinco regimes de desbaste.

Table 5 - Average VPB of wood multi-products from loblolly pine stands growing under five initial plant spacing and five thinning regimes.

( R\$/ha)

\begin{tabular}{clc}
\hline $\begin{array}{c}\text { Espaçamento } \\
\text { Spacing }\end{array}$ & \multicolumn{1}{c}{$\begin{array}{c}\text { Regime de Desbaste } \\
\text { Thinning regime }\end{array}$} \\
\hline$(2-2,5 \times 2,0 \mathrm{~m}) 3.324,24 \mathrm{a}$ & $(2-\mathrm{CF} 20 \mathrm{~s} / \mathrm{desb})$ & $3.337,66 \mathrm{a}$ \\
$(1-2,5 \times 1,2 \mathrm{~m}) 3.238,84 \mathrm{a}$ & $(5-\mathrm{Sist}+\mathrm{Sel} 9$, Sel 15 + CF 20) $3.287,41 \mathrm{a}$ \\
$(3-2,5 \times 2,8 \mathrm{~m}) 3.191,69 \mathrm{ab}$ & $(4-$ Sel 9, Sel 15 + CF 20) & $3.245,87 \mathrm{a}$ \\
$(4-2,5 \times 3,6 \mathrm{~m}) 3.064,89 \mathrm{bc}$ & $(3-$ Sist $9+\mathrm{CF} 20)$ & $2.940,46 \mathrm{~b}$ \\
$(5-2,5 \times 4,4 \mathrm{~m}) 2.931,60 \quad \mathrm{c}$ & $(1-\mathrm{CF} 15 \mathrm{~s} / \mathrm{desb})$ & $2.939,85 \mathrm{~b}$ \\
\hline
\end{tabular}

Observa-se, na Tabela 5, que os espaçamentos mais densos (2,5 x 1,2 m e 2,5 x 2,0 m) produziram um maior VPB, porém não distintos do espaçamento $2,5 \times 2,8 \mathrm{~m}$ a de $5 \%$. Os espaçamentos mais amplos apresentaram médias menores, diferentes estatisticamente.

Considerando os regimes de desbaste, observa-se que os regimes 2 (CF $20 \mathrm{~s} /$ desb), 5 (Sist + Sel 9, Sel 15 + CF 20) e 4 (Sel 9, Sel 15 + CF 20) apresentam as maiores médias, não diferindo significativamente um do outro. Os regimes 1 (CF 15 s/ desb) e 3 (Sist 9 + CF 20) apresentaram médias de VPB estatisticamente menores.

Portanto, a análise isolada dos fatores mostrou que maior renda bruta seria obtida pela venda da madeira advinda de povoamentos plantados em espaçamentos mais densos e cortados aos 20 anos, sem desbaste ou envolvendo desbastes aos 9 e aos 15 anos. Estes resultados corroboram aqueles reportados por Ahrens (1992) e Acerbi et al. (1999), que indicaram regimes parecidos com os aqui eleitos como melhores. Também coincidem com as argumentações de Smith e Strub (1991), que afirmam que para a produção múltipla, espaçamentos menores combinados com desbastes freqüentes podem ser vantajosos.

Apesar de aparentemente clara esta situação, deve-se levar em conta que a interação entre os dois fatores estudados é significativa, o que necessariamente força a analisar os desdobramentos entre espaçamentos e regime de desbaste.

Médias seguidas de mesma letra não diferem entre si pelo teste de Tukey ao nível de 5\% de probabilidade.

a) Comportamento dos espaçamentos dentro de cada regime de desbaste

A Tabela 6 mostra os resultados dos desdobramentos. Observa-se que os cinco espaçamentos têm efeitos diferentes $(\mathrm{p}<0,01)$ sobre todos os regimes de desbaste. As médias dos espaçamentos foram então comparadas pelo teste de Tukey a $5 \%$ de probabilidade, dentro de cada regime de desbaste (TABELA 7). 
TABELA 6 - Desdobramento da interação espaçamento x desbaste para avaliar o comportamento dos espaçamentos dentro de cada regime de desbaste, para a variável VPB.

Table 6 - Analysis of the interaction spacing $\mathrm{x}$ management regime for multi-products gross profit of a loblolly pine experimental stand growing under five initial plant spacing and five thinning regimes.

\begin{tabular}{lcc}
\hline \multicolumn{1}{c}{$\begin{array}{l}\text { Fonte de Variação } \\
\text { Source of variation }\end{array}$} & $\begin{array}{c}\text { Graus } \\
\text { de Liberdade }\end{array}$ & $\begin{array}{c}\text { Quadrados Médios do VPB } \\
\left(\mathrm{m}^{3} / \mathrm{ha}\right)\end{array}$ \\
Espaçamentos dentro de CF 15 s/desb & $d f$ & $429.047,98^{* *}$ \\
Espaçamentos dentro de CF 20 s/desb & 1 & $683.929,49^{* *}$ \\
Espaçamentos dentro de Sist 9+CF 20 & 1 & $805.778,76^{* *}$ \\
Espaçamentos dentro de Sel 9, Sel 15+CF 20 & 1 & $1.875 .476,30^{* *}$ \\
Espaçamentos dentro de Sist+Sel 9, Sel 15+CF 20 & 1 & $3.369 .625,40^{* *}$ \\
Resíduo & 1 & 367,96 \\
\hline
\end{tabular}

** Significativo ao nível de $1 \%$ de probabilidade $(p<0.01)$

TABELA 7 - Desdobramento da interação espaçamento x manejo para avaliar o comportamento dos regimes de desbaste dentro de cada espaçamento, para a variável VPB em povoamentos experimentais de Pinus taeda.

Table 7 - Analysis of the interaction thinning regime $\mathrm{x}$ spacing forgross profit per hectare of a loblolly pine experimental stand growing under five initial plant spacing and five thinning regimes.

\begin{tabular}{lcc}
\hline & Graus & Quadrados Médios do VPB \\
Fonte de Variação & de Liberdade & $\left(\mathrm{m}^{3} / \mathrm{ha}\right)$ \\
Source of variation & $d f$ & Mean squared \\
\hline Desbastes dentro do Espaçamento $2,5 \times 1,2 \mathrm{~m}$ & 1 & $5.389 .874,09^{* *}$ \\
Desbastes dentro do Espaçamento $2,5 \times 2,0 \mathrm{~m}$ & 1 & $1.122 .906,00^{* *}$ \\
Desbastes dentro do Espaçamento $2,5 \times 2,8 \mathrm{~m}$ & 1 & $821.950,72^{* *}$ \\
Desbastes dentro do Espaçamento 2,5 x 3,6 m & 1 & $789.475,01^{* *}$ \\
Desbastes dentro do Espaçamento 2,5 x 4,4 m & 1 & $732.532,71^{* *}$ \\
Resíduo & 120 & 367,96 \\
\hline
\end{tabular}

**Significativo ao nível de $1 \%$ de probabilidade 
Primeiramente, constata-se que os regimes de desbaste 1 (CF 15 s/desb) e 3 (Sist + CF 20) apresentaram menores valores de VPB em todos os espaçamentos avaliados, quando comparados com os outros regimes de desbaste. Nesses dois regimes de desbaste, as diferenças, em termos de VPB, entre os espaçamentos de plantio são pouco marcantes, ficando aquém de $\mathrm{R} \$ 400,00 /$ ha. Aspecto semelhante é constatado para o regime 2 (CF $20 \mathrm{~s} /$ desb).

Nos demais regimes de desbaste, os valores médios de VPB alteraram-se de forma mais expressiva em função do espaçamento de plantio adotado. Tanto no regime 4 (Sel 9, Sel $15+$ CF 20) como no 5 (Sist+Sel 9, Sel $15+$ CF 20), houve tendência de aumento do VPB com o aumento da densidade, porém diferenças inexpressivas foram observadas nos três espaçamentos mais amplos. No regime de desbaste 4, verificou-se diferença significativa entre os dois espaçamentos menores, o que não foi o caso no regime de desbaste 5. Isto implica que se a opção for por desbastes seletivos apenas, pode-se optar por qualquer um dos dois espaçamentos menores (2,5 x 1,2 m ou 2,5 x 2,0 $\mathrm{m})$, sem maiores reflexos no VPB. Ao contrário, se a primeira intervenção prever desbaste sistemáti- co, será mais interessante utilizar o espaçamento $2,5 \times 1,2 \mathrm{~m}$.

Assim, em síntese, à medida que os desbastes são processados de forma mais intensa (maior número de desbastes), a diferença entre os espaçamentos passa a ser mais notória em termos estatísticos. Ou seja, quanto mais denso o plantio inicial, maior será o VPB, no caso de se adotar regimes de desbaste mais intensivos. Ao contrário, com a ausência de desbastes ou a adoção apenas de um único desbaste, não faz muita diferença utilizar espaçamentos mais amplos ou mais densos. Estes fatos mostram a importância de proceder análise experimental da seleção dos espaçamentos e dos regimes de desbaste.

b) Comportamento dos regimes de desbaste dentro de cada espaçamento

A Tabela 8 mostra os resultados dos desdobramentos para os regimes de desbaste dentro de cada espaçamento. Observa-se que os cinco regimes de desbaste têm efeitos diferentes $(\mathrm{p}<0,01)$ sobre todos os espaçamentos. As médias dos espaçamentos foram então comparadas pelo teste de Tukey a $5 \%$ de probabilidade, dentro de cada tipo de desbaste (TABELA 9). 
TABELA 8 - Médias do VPB em povoamentos experimentais de Pinus taeda sob cinco diferentes espaçamentos dentro de cada regime de desbaste.

Table 8 - Avarege gross profit per hectare of a loblolly pine experimental stand growing under thinning regimes.

\begin{tabular}{|c|c|}
\hline $\begin{array}{l}\text { Regime de Desbaste } \\
\text { Thinning Regime }\end{array}$ & $\begin{array}{c}\mathrm{VB}(\mathrm{R} \$ / \mathrm{ha}) \\
\text { Gross Profit }(R \$ / h a)\end{array}$ \\
\hline (1) CF $15 \mathrm{~s} / \mathrm{desb}$ & $\begin{array}{l}(1-2,5 \times 1,2 \mathrm{~m}) 3.090,09 \mathrm{a} \\
(2-2,5 \times 2,0 \mathrm{~m}) 3.002,49 \mathrm{ab} \\
(3-2,5 \times 2,8 \mathrm{~m}) 2.990,24 \mathrm{ab} \\
(4-2,5 \times 3,6 \mathrm{~m}) 2.870,73 \mathrm{ab} \\
(5-2,5 \times 4,4 \mathrm{~m}) 2.745,70 \mathrm{~b}\end{array}$ \\
\hline (2) $\mathrm{CF} 20 \mathrm{~s} / \mathrm{desb}$ & $\begin{array}{l}(2-2,5 \times 2,0 \mathrm{~m}) 3.506,35 \mathrm{a} \\
(3-2,5 \times 2,8 \mathrm{~m}) 3.484,53 \mathrm{a} \\
(4-2,5 \times 3,6 \mathrm{~m}) 3.362,71 \mathrm{ab} \\
(5-2,5 \times 4,4 \mathrm{~m}) 3.219,02 \mathrm{ab} \\
(1-2,5 \times 1,2 \mathrm{~m}) 3.115,71 \mathrm{~b}\end{array}$ \\
\hline (3) Sist $9+$ CF 20 & $\begin{array}{l}(3-2,5 \times 2,8 \mathrm{~m}) 3.089,35 \mathrm{a} \\
(2-2,5 \times 2,0 \mathrm{~m}) 3.087,01 \mathrm{a} \\
(4-2,5 \times 3,6 \mathrm{~m}) 3.003,61 \mathrm{a} \\
(5-2,5 \times 4,4 \mathrm{~m}) 2.866,92 \mathrm{ab} \\
(1-2,5 \times 1,2 \mathrm{~m}) 2.655,41 \mathrm{~b}\end{array}$ \\
\hline (4) Sel 9, Sel 15 + CF 20 & $\begin{array}{l}(1-2,5 \times 1,2 \mathrm{~m}) 3.564,77 \mathrm{a} \\
(2-2,5 \times 2,0 \mathrm{~m}) 3.491,74 \mathrm{ab} \\
(3-2,5 \times 2,8 \mathrm{~m}) 3.204,97 \mathrm{bc} \\
(4-2,5 \times 3,6 \mathrm{~m}) 3.059,95 \mathrm{c} \\
(5-2,5 \times 4,4 \mathrm{~m}) 2.907,95 \mathrm{c}\end{array}$ \\
\hline (5) Sist+Sel 9, Sel $15+$ CF 20 & $\begin{array}{l}(1-2,5 \times 1,2 \mathrm{~m}) 3.855,82 \mathrm{a} \\
(2-2,5 \times 2,0 \mathrm{~m}) 3.446,02 \mathrm{~b} \\
(3-2,5 \times 2,8 \mathrm{~m}) 3.189,36 \mathrm{bc} \\
(4-2,5 \times 3,6 \mathrm{~m}) 3.027,43 \mathrm{c} \\
(5-2,5 \times 4,4 \mathrm{~m}) 2.918,43 \mathrm{c}\end{array}$ \\
\hline
\end{tabular}

Médias seguidas de mesma letra não diferem entre si pelo teste de Tukey ao nível de 5\% de probabilidade. 
TABELA 9 - Médias do volume de madeira com casca de Pinus taeda, para celulose, nos cinco diferentes regimes de desbaste dentro de cada espaçamento.

Table 9 - Average inbark volume of round-wood for pulp and paper of a loblolly pine experimental stand growing under different initial spacing.

\begin{tabular}{|c|c|c|c|}
\hline & Espaçamento & $\overline{\mathrm{VPB}}(\mathrm{R}$ & $\$ /$ ha) \\
\hline Spacing & & Gross Profi & $i(R \$ / h a)$ \\
\hline & (1) $2,5 \times 1,2 \mathrm{~m}$ & $(5-$ Sist+Sel 9, Sel 15 + CF 20) & $3.855,82 \mathrm{a}$ \\
\hline & & $(4-$ Sel 9, Sel $15+$ CF 20$)$ & $3.564,77 \mathrm{a}$ \\
\hline & & $(2-\mathrm{CF} 20 \mathrm{~s} / \mathrm{desb})$ & $3.115,71 \mathrm{~b}$ \\
\hline & & (1 - CF $15 \mathrm{~s} / \mathrm{desb})$ & $3.002,49 \mathrm{~b}$ \\
\hline & & (3 - Sist $9+$ CF 20) & $2.655,41 \quad \mathrm{c}$ \\
\hline & (2) $2,5 \times 2,0 \mathrm{~m}$ & $(2-\mathrm{CF} 20 \mathrm{~s} / \mathrm{desb})$ & $3.506,35 \mathrm{a}$ \\
\hline & & $(4-$ Sel 9, Sel $15+$ CF 20$)$ & $3.491,74 \mathrm{a}$ \\
\hline & & (5-Sist+Sel 9, Sel 15 + CF 20) & $3.446,02 \mathrm{a}$ \\
\hline & & (1 - CF $15 \mathrm{~s} / \mathrm{desb})$ & $3.090,09 \mathrm{~b}$ \\
\hline & & $(3-$ Sist $9+$ CF 20) & $3.087,01 \quad b$ \\
\hline & (3) $2,5 \times 2,8 \mathrm{~m}$ & $(2-\mathrm{CF} 20 \mathrm{~s} / \mathrm{desb})$ & $3.484,53 \mathrm{a}$ \\
\hline & & $(4-$ Sel 9, Sel $15+$ CF 20$)$ & $3.204,97 \mathrm{ab}$ \\
\hline & & (5-Sist+Sel 9, Sel 15 + CF 20) & $3.189,36 \mathrm{ab}$ \\
\hline & & (3- Sist $9+$ CF 20) & $3.089,35 \mathrm{~b}$ \\
\hline & & (1 - CF $15 \mathrm{~s} / \mathrm{desb})$ & $2.990,24 \quad b$ \\
\hline & (4) $2,5 \times 3,6 \mathrm{~m}$ & $(2-\mathrm{CF} 20 \mathrm{~s} / \mathrm{desb})$ & $3.362,71 \mathrm{a}$ \\
\hline & & $(4-$ Sel 9, Sel $15+$ CF 20$)$ & $3.059,95 \mathrm{ab}$ \\
\hline & & (5-Sist+Sel 9, Sel 15 + CF 20) & $3.027,43 \mathrm{ab}$ \\
\hline & & (3 - Sist $9+$ CF 20) & $3.003,61 \mathrm{~b}$ \\
\hline & & (1 - CF $15 \mathrm{~s} / \mathrm{desb})$ & $2.870,73 \mathrm{~b}$ \\
\hline & (5) $2,5 \times 4,4 \mathrm{~m}$ & $(2-\mathrm{CF} 20 \mathrm{~s} / \mathrm{desb})$ & $3.219,02 \mathrm{a}$ \\
\hline & & (5-Sist+Sel 9, Sel 15 + CF 20) & $2.918,43 \mathrm{ab}$ \\
\hline & & $(4-$ Sel 9, Sel $15+$ CF 20$)$ & $2.907,95 \mathrm{ab}$ \\
\hline & & (3 - Sist $9+$ CF 20) & $2.866,92 \mathrm{~b}$ \\
\hline & & (1 - CF $15 \mathrm{~s} / \mathrm{desb})$ & $2.745,70 \quad b$ \\
\hline
\end{tabular}

Médias seguidas de mesma letra não diferem entre si pelo teste de Tukey ao nível de 5\% de probabilidade. 
Conforme pode ser observado na Tabela 9, no espaçamento mais denso $(2,5 \times 1,2 \mathrm{~m})$, os regimes de desbaste com maior número de intervenções foram aqueles que preponderaram como melhores (regimes 5 e 4). 0 tratamento que apresentou o menor valor de VPB foi o regime de desbaste 3 (Sist 9 + CF 20). Os regimes sem desbaste ficaram colocados em posição intermediária, não diferindo um do outro.

Para o espaçamento 2 (2,5 x 2,0 m) não foram evidenciadas diferenças entre os regimes de desbaste 2, 4 e 5, os quais mostraram-se diferentes dos demais (regimes 1 e 3), que por sua vez se apresentaram iguais do ponto de vista experimental.

Os regimes de desbaste 3, 4 e 5 apresentaram padrões semelhantes, isto é, maiores médias para os regimes de desbaste 2,4 e 5 , porém sem diferença estatística entre os regimes 4, 5, 3 e 1. Nesses regimes, as diferenças entre densidades são de menor magnitude. Isto implica que, em espaçamentos mais amplos que 2,5 x 2,8 m, o regime de desbaste não provoca alteração relevante no VPB. Ao contrário, nos espaçamentos mais densos, o efeito dos desbastes mostra-se importante no aumento da renda bruta.

Em síntese, a maior média de VPB foi encontrada para a combinação entre o espaçamento $1(2,5 \times 1,2 \mathrm{~m})$ com o regime de desbaste 5 (Sist+Sel 9, Sel 15 + CF 20). Todavia, a opção por não utilizar o desbaste sistemático também é igualmente uma opção viável (regime 4). Se a opção for pelo regime de desbaste 4 , então, pode-se até cogitar a adoção do espaçamento 2,5 x 2,0 m, sem perda significativa de retorno financeiro. Outra opção que pode ser também estudada com atenção é a combinação do espaçamento 2,5 x 2,8 m com o regime com corte final aos 20 anos sem desbaste. Esta opção tem se mostrado interessante em pesquisas que consideram exclusiva madeira para laminação e serraria por Sanquetta et al. (no prelo, a,b).

\section{Conclusões}

Os resultados desta pesquisa indicam que, em termos de renda bruta advinda da venda de múltiplos produtos de madeira, a combinação ideal é o emprego de um espaçamento de plantio menor, isto é, povoamento mais denso, como o $2,5 \times 1,2 \mathrm{~m}$, combinado com a realização de des- bastes mais freqüentes, aos 9 e aos 15 anos, preferivelmente com a adoção de desbaste sistemático na primeira intervenção. Essa combinação determinou a maior renda bruta no presente estudo. Contudo, diversas outras opções podem ser igualmente interessantes, inclusive a adoção de um espaçamento intermediário combinado com um regime sem desbaste. Isto revela a importância de proceder a análise experimental dos regimes de manejo a serem selecionados, permitindo, assim, decidir com melhor juízo a opção que gera a maior renda bruta.

Neste trabalho foram consideradas apenas as rendas brutas dos diferentes tratamentos de espaçamento e desbaste, não sendo levados em conta os respectivos custos. Estudos complementares poderão clarificar a influência que os custos exercem sobre a definição da melhor combinação de densidade de plantio e regime de desbaste.

\section{Referências}

ACERBI Jr., F. W. et al. Simulação e avaliação econômica de desbastes para Pinus taeda para obtenção de múltiplos produtos da madeira. Cerne, Lavras, v. 5, n.1, p. 81-102, 1999.

ACERBI Jr., F. Definição de regimes ótimos de desbaste e poda em Pinus ta edh., 1998. Dissertação (Mestrado) - Universidade Federal de Lavras, Lavras, 1998.

AHRENS, S. A seleção simultânea do ótimo regime de desbastes e da idade de rotação, para povoamentos de Pinus taed modelo de programação dinâmica. 1992. $189 \mathrm{f}$. Tese (Doutorado) - Universidade Federal do Paraná, Curitiba, 1992.

DRAPPER, N. R.; SMITH, H. Applied regression analysis. 2. ed. New York: John Wiley \& Sons, 1980. p. 709.

GOMES, F. S; MAESTRI, R; SANQUETTA, C. R. Análise financeira de regimes de desbaste em povoamentos de Pinus taeda L, visando a produção de madeira para a indústria de papel e celulose. Revista Árvore, Viçosa, v. 22, n. 2, p. 227-243, 1998.

GOMES, F. S. A Seleção de regimes de desbaste mais rentáveis em Pinus taed 
de madeira para papel e celulose. 1999. $140 \mathrm{f}$. Dissertação (Mestrado) - Universidade Federal do Paraná, Curitiba, 1999.

OLIVEIRA, E. B. Um sistema computadorizado para prognose do crescimento e produção de Pinus ta edb., com critérios quantitativos para avaliação técnica e econômica de regimes de desbaste. 1995. 134 f. Tese (Doutorado) - Universidade Federal do Paraná, Curitiba, 1995.

OLIVEIRA, E. B.; MACHADO, S. A.; FILHO, A. F. Sistema para simulação do crescimento e da produção de Pinus taeda L e a avaliação econômica de regimes de desbaste. Revista Árvore, Viçosa, v. 22, n.1, p. 99-111, 1998.

SCOLFORO, J. R. S.; MACHADO, S. A. Um sistema de crescimento e produção com simulador de desbaste. Scientia Forestalis, Piracicaba, n. 50, p. 51-64, 1996.

SCOLFORO, J. R. S.; MAESTRI, R. O manejo de florestas plantadas. In: Manejo Florestal. Lavras: UFLA/FAEPE, 1998. p. 315 -379; 443.

SHEPHERD, K. R. Plantation Silviculture: Dordrecht. Martinus Nijhoff, 1986. p. 322.

SMITH, W. D.; STRUB, M. R. Inicial spating: how many trees to plant. In: Forest regeneration manual. Dordrecht: Keuwer Ac., p. 281-289. 1991.
SOKAL, R. R; ROHLF, F.J. Biometry: The principles and practice of statistics in biological research. New York: Freeman, 1981. p. 859.

VOLPI, N. M. P. 0 impacto de perturbações estocásticas em um modelo de planejamento florestal. 1997. 268 f. Tese (Doutorado) - Universidade Federal do Paraná, Curitiba, 1997.

\section{Agradecimentos}

Os autores deste artigo agradecem à empresa Pisa Florestal S.A., que gentilmente cedeu os dados usados nesta pesquisa e ao pesquisador da Embrapa Florestas, Dr. Edilson Batista de Oliveira, que forneceu uma cópia do programa computacional SISPINUS para emprego neste trabalho. 